\title{
La Crusca risponde ogni tre per due o ogni due per tre?
}

\author{
Matilde Paoli
}

PUBBLICATO: 13 MARZO 2020

\section{Quesito:}

Molti lettori ci scrivono, specialmente dal Nord Italia, ma anche da Firenze, Roma e Sassari, chiedendo chiarimenti su un modo di dire "numerico": per significare che qualcosa accade molto spesso si dice che avviene ogni tre per due oppure ogni due per tre? Qual è l'origine di questa espressione? È un modo di dire diffuso da sempre su tutto il territorio nazionale o si è originato in una particolare area?

\section{La Crusca risponde ogni tre per due o ogni due per tre?}

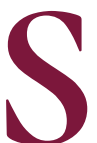

icuramente queste espressioni suscitano molta curiosità e non solo nei nostri lettori, visto che da un'interrogazione tramite Google vediamo che molte delle pagine in cui ricorrono le I27.00o occorrenze in italiano di "ogni tre per due" e le 389.000 di "ogni due per tre" (dati al 2/I2/2019) ospitano discussioni su quale sia la loro origine o su quale, tra le due, debba considerarsi la versione "corretta".

Qualcuno pensa che si tratti di espressioni recenti; qualcuno collega la forma ogni tre per due alle offerte del supermercato; altri (cosi i curatori della rubrica Scioglilingua del "Corriere della Sera" 9/1/2010) considerano primaria la sequenza ogni due per tre e la interpretano come "due volte su tre" e ritengono che l'offerta del supermercato potrebbe essere eventualmente alla base del "rovesciamento" del modo.

Da quel che possiamo dedurre dalla rete, le espressioni sembrano comuni al Nord (sicuramente in Piemonte, Lombardia ed Emilia-Romagna), ma anche al Centro, Toscana compresa (in cui pare prevalere ogni due per tre) e anche al Sud, specie in Sicilia. Della diffusione territoriale e della supposta spartizione geografica della penisola tra le due versioni si è occupata anche Carla Bazzanella in Numeri per parlare: Da 'quattro chiacchiere' a 'grazie mille' (Gius.Laterza \& Figli Spa, 20II, ediz. digitale 20I4) dove si legge:

Con il significato di 'molto spesso', in italiano ritroviamo il due collegato al numero che lo segue: ogni due per tre è un modo di dire diffuso non solo in area settentrionale. Alcuni dati soggettivi, ricavati da un post in un blog, sembrano confermarlo:

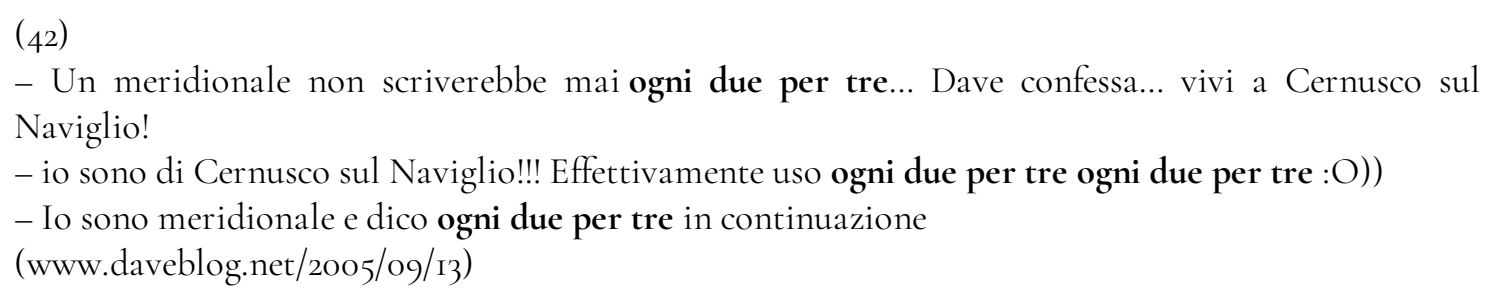

Qualunque sia la versione originale, il modo sembra approdato in italiano comune piuttosto tardi; per quanto riguarda la stampa, troviamo su archive.org una testimonianza di ogni tre per due (ed è la prima per il sito) sull'“Unità" del Io/6/I995: "gli Italiani sono stati chiamati alle urne ogni tre per due". L'espressione non trova risultati nell'archivio della "Repubblica", mentre ogni due per tre ne trova 9i e 
anche in questo caso la prima occorrenza risale al 1995: "...la sua [di Buttiglione] voglia di fare passi indietro e riflettere un po', il fascino indiscreto del diventare a pieno titolo un uomo di centro quale lui si definisce ogni due per tre" (Barbara Palombelli, La rotta a zig zag del capitano bianco, 22/2/1995).

Rintracciamo un'attestazione un po' meno recente di ogni due per tre in Google Libri (dove non si trovano attestazioni pertinenti di ogni tre per due prima del secolo attuale), nella traduzione italiana di Los pájaros de Bangkok di Manuel Vázquez Montalbán (Gli uccelli di Bangkok, Feltrinelli, 1990):

Ho un'ex moglie sposata con un cattedratico ricchissimo che porta molto bene il frac e lo mettono ogni due per tre su 'Jours de France'. (p. 268)

Nell'edizione originale del 1983 l'espressione spagnola è letteralmente identica a quella italiana nella forma e nel significato:

Tengo a mi ex mujer casada con un catedrático riquísimo al que le sienta muy bien el frac y le sacan cada dos por tres en Jours de France. (p. 225)

\section{In spagnolo}

In lingua spagnola il modo, in entrambe le varianti, sembra molto diffuso: da una ricerca su Google di "cada dos por tres" (dati al 2/I2/2019) le pagine in spagnolo provenienti dalla Spagna sono 557.00o, mentre quelle provenienti da tutti i paesi sono r.650.00o; alle stesse condizioni "cada tres por dos" trova rispettivamente 19. Ioo e 82.800 occorrenze. In alcuni casi l'espressione è introdotta dalla preposizione á (o anche $a$ ) con occorrenze notevolmente minori.

Le prime attestazioni di (á) cada dos por tres in Google Libri risultano in due testi editi in Spagna nel XIX secolo (Napoleon; ó El verdadero D. Quixote de la Europa [...], I8I3 e La Iglesia Romana y la revolución, di Jacques-Augustin-Marie Crétineau-Joly, "traducida al castellano de la tercera edition francesa" da D. Victor Gebhardt, I867, in cui traduce l'originale "à tous moments"). In entrambi i casi, la locuzione è introdotta dalla preposizione e ha lo stesso significato di quella italiana. Anche le primissime testimonianze del secolo successivo hanno la preposizione, mentre ne sono prive quelle più tarde, come le quattro reperibili nell'opera teatrale El financista dell'argentino Felix M. Pelayo del I947 .

$(A)$ cada tres por dos non ha attestazioni nel XIX secolo e una soltanto in quello successivo, praticamente sulle soglie del XXI, senza preposizione, nell'epistolario di Rocío Espada, Mis Cartas a María, Resumen de Las Célebres Cartas de Noverre Sobre Las Danzas Y El Ballet (I997).

Nel XXI secolo le testimonianze di cada dos por tres senza la preposizione introduttiva sono in netta maggioranza e delle poche decine di attestazioni della variante a numeri invertiti solo due hanno la preposizione.

A proposito della coesistenza delle due varianti ci sembra interessante notare che nella lunga lista di parole e modi di dire tipici dello spagnolo di Argentina confrontati con gli equivalenti castigliani che Alejandro Placer riporta in Los argentinos hablan español? (2003) troviamo Cada tres por dos a fronte del castigliano Cada dos por tres. Una libera alternanza mostra invece il più recente Escalera al cielo (2015), opera destinata al pubblico infantile dello scrittore messicano Andrés Acosta:

Tropezabas á cada dos por tres y á cada tres por dos, hasta que tu mamá te las quitó para arrojarlas a la basura. ['inciampavi ogni due per tre e ogni tre per due, finché tua madre te li tolse (ndr: si parla di una sorta di paraocchi) per gettarli nell'immondizia'.] 
Da questa progressione interna allo spagnolo sembrerebbe che la versione primaria sia á cada dos por tres, che con il passar del tempo ha perduto la preposizione introduttiva e ha generato una versione con i numeri invertiti diffusasi in particolare, ma non solo, al di fuori del territorio europeo.

\section{Le possibili origini}

In lingua spagnola esiste l'espressione á dos por tres che ha una lunga tradizione nella lessicografia. Nel Diccionario de la lengua castellana en que se explica el verdadero de las voces etc... della Real Academia Española edito da Francisco del Hierro (I726-1739) la locuzione è registrata con due accezioni, una relativa a un modo di parlare, l'altra a un modo di agire: 'modo di parlare che si usa quando una persona esprime a un'altra il suo pensiero e il suo sentire in modo chiaro e senza costrizioni.[...] Vale anche lo stesso che senza timore, senza ritegno' (traduzione mia). A sostegno della prima accezione si riporta una citazione - "El vergantón le dixo à dos por tres que mentía" - tratta dal Cuento de cuentos di Francisco de Quevedo y Villegas (I580-I645), mentre per la seconda si cita un passo dalle Novelas ejemplares di Cervantes (riporto un contesto più ampio di quello presente nel dizionario, per la fonte v. oltre):

Pero ninguna cosa me admiraba más, ni me parecía peor, que il ver que estos jiferos con la misma facilidad matan a un hombre que a una vaca: por quítame allá esa paja, á dos por tres meten un cuchillo de cachas amarillas por la barriga de una persona come si acocotasen un toro. (La de los perros Cipión y Berganza ['La dei cani Scipione e Berganza'])

A proposito di questo passo abbiamo una testimonianza significativa nell'Edizione complanare del testo spagnolo e delle versioni di G. A. Novilieri Clavelli e D. Fontana (resa disponibile in rete dall'Università di Padova), che mette a fronte il testo spagnolo delle Novelas ejemplares nell'edizione di Madrid del I6Iz e le prime traduzioni italiane integrali di poco successive all'originale, la versione "del francese italianizzante" Guglielmo Alessandro de Novilieri Clavelli (Guillaume Alexandre de Noviliers Clavel) del 1626 e quella di Donato Fontana del 1627 . Le due versioni traducono á dos por tres in modo differente dall'originale e anche tra di loro, il che fa supporre che l'italiano dell'epoca non disponesse di una locuzione equivalente nella forma. La traduzione di de Novilieri Clavelli ricorre a una locuzione, "in un voltar di mano", totalmente diversa da quella spagnola e che ha valore temporale: si tratta di un presumibile calco sul francese en un tour de main, risalente al XVI secolo, che vale "dans le temps qu'il faut pour tourner la main, très rapidement" (cfr. Atilf s.v. tournemain).

La scelta del Fontana, "alle due o alle tre", più simile a quella spagnola con la quale ha in comune i valori numerici, sembra riferirsi a indicazioni orarie.

Di questa espressione abbiamo trovato una sola testimonianza nella Fuga amorosa comedia di Biagio Micalori da Urbino (I62I); nel passo, almeno stando alla lettera, difficilmente la locuzione potrebbe essere intesa come equivalente di in un voltar di mano, ma piuttosto parrebbe analoga a prima o poi o anche molto spesso, di frequente.

Emi[lia]: [...] non ci è cosa nondimeno, che più ammollisca un cuore, quanto le lagrime, et il pianto di bella donna.

Ros[etta]: E per questo vedete, che le donne non ne fan carestia; che alle due, o alle tre gettano fuori certe lagrimone, che pajono nocciuole... (atto II, scena VII)

Ciò che sembra accomunare le due versioni è l'interpretazione in senso temporale della locuzione spagnola á dos por tres. In questa direzione sembra andare anche l'edizione ottocentesca del Diccionario de la lengua castellana por la Academia española, che riferisce l'espressione a un atteggiamento, un modo di agire o di parlare, sottolineandone però la rapidità, almeno nel caso del modo di parlare. 
á dos por tres. Modo de hablar que se usa para expresar que alguno dice su parecer con demasiada prontitud, ó hace alguna cosa sin miedo ni reparo. ['modo di parlare che si usa per indicare che qualcuno esprime la sua opinione con troppa prontezza, o agisce senza timore, né ritegno'] (7. ed., Madrid, Impr. real, i832 s.v. dos)

Lo stesso dizionario non registra invece la locuzione (á) cada dos por tres, che pure (come abbiamo visto) cominciava a essere in uso in quegli anni. La troviamo solo nella seconda metà del secolo successivo nel Diccionario enciclopédico Espasa (vol. 5, 1978) che la registra accanto a á dos por tres e a una terza espressione, en un dos por tres. Le rispettive descrizioni sono le stesse che troviamo s.v. dos nella Actualización 2018 del Diccionario della RAE: á dos por tres, etichettato come colloquiale, vale "Pronta y claramente", en un dos por tres, ancora indicato come colloquiale, vale "En un momento, rápidamente" e (á) cada dos por tres "Con frecuencia".

I significati delle tre espressioni non sono poi cosi distanti: si prende avvio da un modo di parlare o di agire (á dos por tres) che vale 'prontamente e in modo chiaro', in cui troviamo anche una sfumatura temporale in pronta = prontamente; si passa a en un dos por tres in cui l'aspetto temporale è prevalente, 'in un momento, rapidamente'; per arrivare a un ripetersi continuo di quel modo momentaneo: da 'in un momento' a 'a ogni momento'. Sembra possibile quindi ipotizzare che il più tardo (á) cada dos por tres sia un'evoluzione delle altre espressioni.

\section{I concorrenti italiani}

Come abbiamo visto il modo (á) cada dos por tres, risultato un progresso interno allo spagnolo, potrebbe essere alla base del modo italiano ogni due per tre / ogni tre per due. Occorre pero considerare che in alcune aree della nostra penisola esistono varianti un po' diverse, radicate nel parlato tradizionale locale, che hanno lo stesso significato.

Per esempio in una discussione in rete a proposito del modo ogni due per tre troviamo questa affermazione:

In Sicilia (ma non so nel resto del... mondo) quando si vuole accusare qualcuno di insistenza fastidiosa si dice che fa una cosa "ogni dui e tri": nun nni pozzo cchiù, mi telefuna ogni dui e tri.

Praticamente ha il senso di "in continuazione"; non si ferma a uno, ma continua due, tre... volte.

Effettivamente nel Vocabolario siciliano etimologico, italiano, e latino, dell'abbate Michele Pasqualino da Palermo (1785) troviamo: "Ad ogni dui tri, posto avverbialm. Vale, spessissimo a ogni piè sospinto, persaepe, saepissime". Pressoché identica l'attestazione del Nuovo dizionario siciliano-italiano di Vincenzo Mortillaro (1838). Anche questa espressione, di molto precedente al calco novecentesco, potrebbe essere attribuita all'influenza spagnola vista la dominazione plurisecolare sull'isola.

Con lo stesso significato esiste un'altra espressione assai simile, ma con l'ordine dei numeri invertito, ogni tre due (anche nelle grafie ogni tre-due e ogni tre/due ) che troviamo in rete in testi riconducibili all'area campana (altra area sottoposta a dominazione spagnola). Eccola ancora in un post calcistico con coloritura dialettale:

Ind a stu forum m parit tanta passaguai: scugnizza ca s fa semp male, barto ca s fa lavaggi, aerosol e ten o cateter, esno ca ten crisi di identità, tonin ca ogni tre due scassa o computer, style che a furia di contare i soldi ha la superficie dell'indice e del pollice lacerate...e che caspita :nono: :look:

(intervento su CalcioNapoliz4 Forum, 28/II/20I3)

Espressione identica stando alle testimonianze della rete è diffusa in alcune aree della Toscana 
orientale (Aretino, Casentino, Valdarno superiore). Ecco un esempio in un post pubblicato su amarantomagazine.it, sito del tifo aretino il II/o3/20I2:

quanta gente che ogni tre-due diceva a martucci adorante:"graaande!!!!"

(commento dell'rı/3/20I2 su Amarantomagazine.it)

La versione tosco-campana ogni tre due potrebbe avere un'origine del tutto indipendente: possiamo infatti interpretarla come "ogni tre (eventi, manifestazioni, oggetti, ecc.) due (hanno la caratteristica x)", ovvero "(la circostanza avere la caratteristica x si verifica) assai spesso". Diverso il caso del modo siciliano, (a) ogni dui e tri, in cui troviamo l'ordine inverso, che è poi quello della progressione numerica.

Inoltre si può notare come i numeri in questione risultino presenti in diversi modi idiomatici (non avere due per fare tre 'mancare del necessario' che troviamo in Lurati 200 I o non c'e due senza tre in Bazzanella, cit.) o espressioni assai frequenti (due o tre, o anche due tre, per indicare un numero scarso, una piccola quantità, ancora in Bazzanella, o anche alle due o alle tre citato sopra). Ciò li rende comunque disponibili per creazioni "autonome" e indipendenti. Difficile dunque dare indicazioni certe sull'origine dei diversi modi basati su queste cifre e sui loro eventuali rapporti reciproci.

Quel che possiamo affermare è che in particolare la locuzione ogni due per tre / ogni tre per due sembra essere penetrata in italiano piuttosto recentemente, nella seconda metà del secolo scorso; che la si deve a un probabile tramite spagnolo la cui forma originaria è á cada dos por tres, modo a sua volta presumibilmente legato alle espressioni più antiche á dos por tres e en un dos por tres. Come in spagnolo anche in italiano la versione più aderente all'originale è da considerarsi quella in cui il 2 precede il 3 , ovvero ogni due per tre.

\section{Cita come:}

Matilde Paoli, La Crusca risponde ogni tre per due o ogni due per tre?, "Italiano digitale", 2019, XII, 2020/1 (gennaio-marzo)

DOI: $10.35948 / 2532-9006 / 2020.3271$

Copyright 2019 Accademia della Crusca

Pubblicato con licenza creative commons CC BY-NC-ND 